\title{
Vacuum Lamination of a Stretchable Sensor System in PolyPropylene
}

\author{
Pieter Bauwens \\ CMST-ELIS \\ imec and Ghent University \\ Ghent, Belgium \\ 0000-0001-9398-2485
}

\author{
Frederick Bossuyt \\ CMST-ELIS \\ imec and Ghent University \\ Ghent, Belgium \\ 0000-0003-3350-9295
}

\author{
Jan Patrick Deckers \\ $V I G O$ \\ Wetteren, Belgium \\ Jan.Deckers@ vigogroup.eu
}

\author{
Jan Vanfleteren \\ CMST-ELIS \\ imec and Ghent University \\ Ghent, Belgium \\ 0000-0002-9654-7304
}

\begin{abstract}
The explosive growth of the so-called Internet-ofThings, where more and more everyday objects are becoming 'smart' and connected, demands for reliable integration technologies for electronics in all kinds of materials. If we want these electronics to be as least intrusive as possible, they preferably conform to the shape of the contemplated object. In this article we want to present a technique that allows the integration of a smart sensor system in a thermoplastic material (polypropylene, PP) by vacuum lamination. This laminated stack can then be thermoformed from a flat sheet into the desired 3D shape. The sensor system in question is a sensor bus incorporating three inertial movement sensors. Each sensor is placed on a separate small, thin $(200 \mu \mathrm{m})$ FR4 PCB together with some necessary peripheral components. These smart sensor nodes are then placed on a flexible, stretchable circuit, which is then laminated between two $2 \mathrm{~mm}$-thick PP sheets. These sheets can subsequently be heated and thermoformed. In this particular case, the PP was used to create a smart ankle-foot orthosis (AFO). Although long-term reliability needs to be improved, we can show that the discussed technology allows for a successful lamination and thermoforming. This paper will elaborate on the sensor system, stretchable bus system and lamination technique, together with the encountered problems and implemented mitigations. While the current application is the aforementioned AFO, the sensor system can easily be expanded to other types of sensors and the thermoforming process allows for a wide range of possible applications.
\end{abstract}

Index Terms-Flexible electronics, sensor system, vacuum lamination, thermoforming, polypropylene

\section{INTRODUCTION}

Thermoforming is an easy an cheap method to transform sheets of thermoplastic material, such as PolyPropylene (PP) into a desired 3D shape. In this paper we describe an approach to embed an active sensor circuit in such a thermoplastic sheet by vacuum lamination. This laminated stack can then be thermoformed, providing an end result as a 'smart' object, ready to be integrated in the world of Internet-of-Things. This approach should be reliable and robust, meaning that the integrated sensor circuit should survive both the lamination and thermoforming process, and remain functional under regular use of the object.

This research was part of the ICON project 'Intelligent Instrumented AnkleFoot Orthosis' (I2AFO), project number HBC.2016.0654, funded by imec and the Flemish government.
While the technology is not limited to a single type of sensor, the technology was developed with an Inertial Measurement Unit (IMU). These sensors are capable of measuring their orientation and are useful to determine the orientation of (individual parts or regions of) our smart object.

In this paper, we describe the general approach of the lamination and subsequent thermoforming process. However, the technology was developed with a specific application in mind, which will be elaborated at the end of this paper. In short, the primary goal was to develop an intelligent AnkleFoot Orthosis (AFO). Each AFO is custom created for a specific customer by thermoforming a PP sheet over a custom mold. An intelligent AFO could help assess efficacy, monitor usage, provide gait analysis, and more.

\section{PROCESS}

\section{A. Goal and Requirements}

We want to develop a reliable technique to integrate a sensor system into a thermoplastic material such as PolyPropylene (PP), to be thermoformed afterwards. This poses some requirements on both our system and our approach.

Our sensor system should be as generic as possible, to open the doors to a whole range of applications. For this reason, we opted to build our sensor system around an $\mathrm{I}^{2} \mathrm{C}$ bus topology. In an $\mathrm{I}^{2} \mathrm{C}$ bus, there is a single controller, or master, that manages the entire bus. Besides this Master there are one or more slaves, who are able to communicate with (and only with) that master. In principle, the $\mathrm{I}^{2} \mathrm{C}$ bus consists of two wires - a data line, SDA, and a clock line, SCL - that connect all slaves to the master (See Figure 1). Each slave is equipped with a unique address, used by the master to initiate communication. In an existing $\mathrm{I}^{2} \mathrm{C}$ bus system, new slaves can be added (and removed) without issue, as long as the new address is unique to the system. Because of the widespread use of the $\mathrm{I}^{2} \mathrm{C}$ protocol, a lot of smart digital sensors already have an $\mathrm{I}^{2} \mathrm{C}$ interface integrated. If not, a 'dumb' analog sensor can easily be equipped with a small microcontroller to create a smart sensor node (see also Section III). With this, we have a sensor system where the senors can be distributed throughout the PP, with just a single connection bus connecting them to the master. Apart from the two communication lines needed for the $\mathrm{I}^{2} \mathrm{C}$ protocol, the bus also includes a voltage supply 


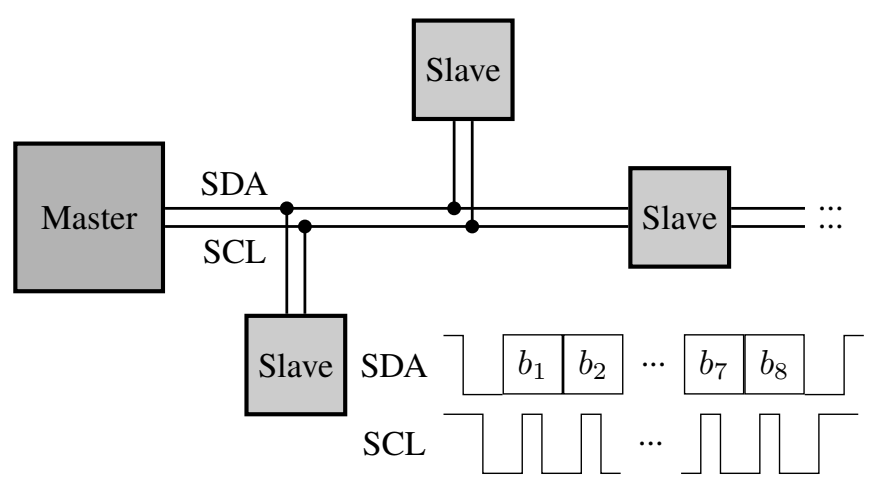

Fig. 1. Schematic representation of an $\mathrm{I}^{2} \mathrm{C}$ sensor system.

and ground line to power the sensor nodes. While the system is not dependent on the type of sensor, the technology was developed with Inertial Movement Units (IMUs) in mind. This IMU (BNO055) measures the orientation of the sensor, based on the integrated accelerometer, magnetometer and gyroscope. Our application demanded for three such sensors over a range of about $30 \mathrm{~cm}$ (see also Section III).

As the thermoforming process will stretch both the plastic as the laminated sensor system, this sensor system by itself should be at least as stretchable as the plastic. After this process and the PP has hardened, further stretching of the system will be minimal. As the $\mathrm{I}^{2} \mathrm{C}$ bus master will also be tasked to communicate with the outside world and will be equipped with a rechargeable battery (Section III), the master itself will not be integrated into the PP, and thus a connector should be provided.

In the final product, we want a stable and reliable connection to all embedded sensors.

\section{B. Thermoforming}

In a thermoforming process, a thermoplastic material (PP in this case) is heated in an oven to a suitable temperature allowing it to be stretched into or over a mold into the desired shape. This stretching can be done mechanically through a vacuum or by manually draping the moldable sheet over the mold (See Figure 2).

While the exact position of the sensors in the final 3D shape is not extremely crucial, we nevertheless needed to examine how the PP sheet is stretched, in order to have at least an idea of the approximate position of the sensors after thermoforming. This would also give us an idea about how much our sensor system should be able to stretch. To achieve this, we equipped a PP sheet with a grid $(5 \mathrm{~mm} \times 5 \mathrm{~mm})$ and measured how this grid was transformed after thermoforming. With our mold (see Section III) the stretching varied between $0 \%$ and about $100 \%$. This transformation grid also allowed us to determine the locations of the sensors on the flat PP sheet, so they'd end up at the desired position after forming.

\section{Lamination stack}

Our application demanded for a $4 \mathrm{~mm}$ thick stack, with the sensor system embedded somewhere in the middle of the stack.

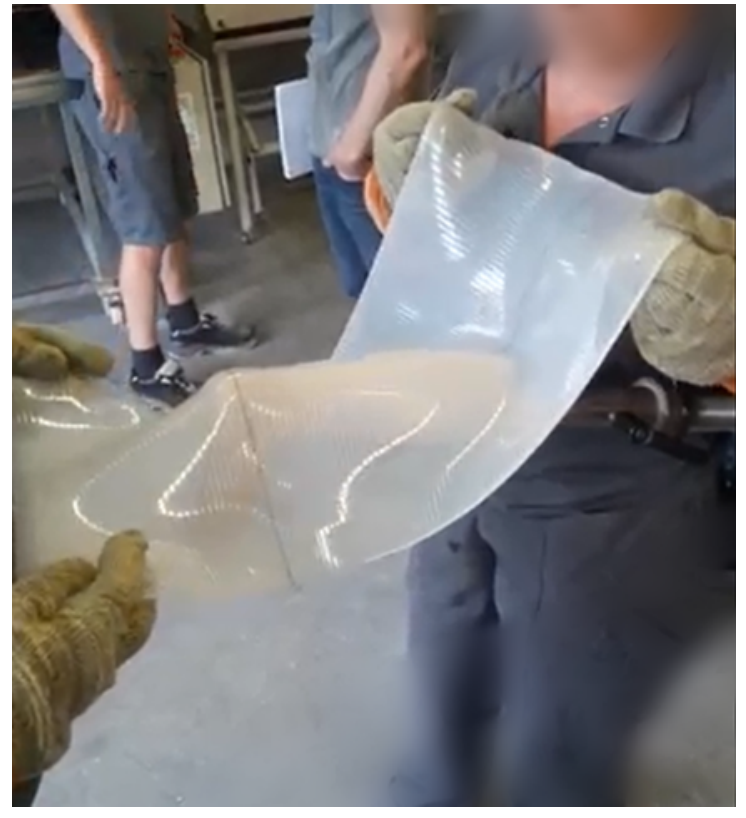

Fig. 2. The heated PP sheet is thermoformed by draping it over a designed mold.

Using a dummy sensor circuit, a couple of stack builds were evaluated:

- PP (2 mm) - Ethylene-Vinyl Acetate (EVA) film - circuit - PP (2 mm)

An EVA film was added between two layers of PP as an adhesion layer, but it was quickly clear that the total stack was too rigid after heating to perform accurate forming.

- PP (2 mm) - EVA-film below circuit - circuit - PP (2 mm)

Only applying the EVA below the circuit improved the plasticity of the stack, but caused the stack to delaminate after cooling.

- PP (2 mm) - circuit - PP (2 mm)

The adhesion between the two PP sheets proved sufficient to keep the circuit in place and didn't show a noticeable difference in the manual thermoforming of the sheet. We opted to use this stack for the further process development.

\section{Initial run}

We designed a flexible $\mathrm{I}^{2} \mathrm{C}$ bus of the appropriate length with three 'islands' to accommodate for the three sensors. The $\mathrm{I}^{2} \mathrm{C}$ bus consists of 4 meandered copper tracks, supported by a polyimide (PI). We start from double sided material AP8525 from Dupont. This material consists of 50 $\mu$ m Kapton polyimide material and $17 \mu \mathrm{m}$ base copper. As the application only requires one layer, the functional image is chemically etched on one side of the flex laminate. The other layer is entirely etched off. Coverlayer of type FR0110 is used which consists out of $25 \mu \mathrm{m}$ adhesive and $25 \mu \mathrm{m}$ Kapton polyimide with an overall thickness of $50 \mu \mathrm{m}$. The coverlayer is laminated selectively where direct soldering is not applicable. As such 


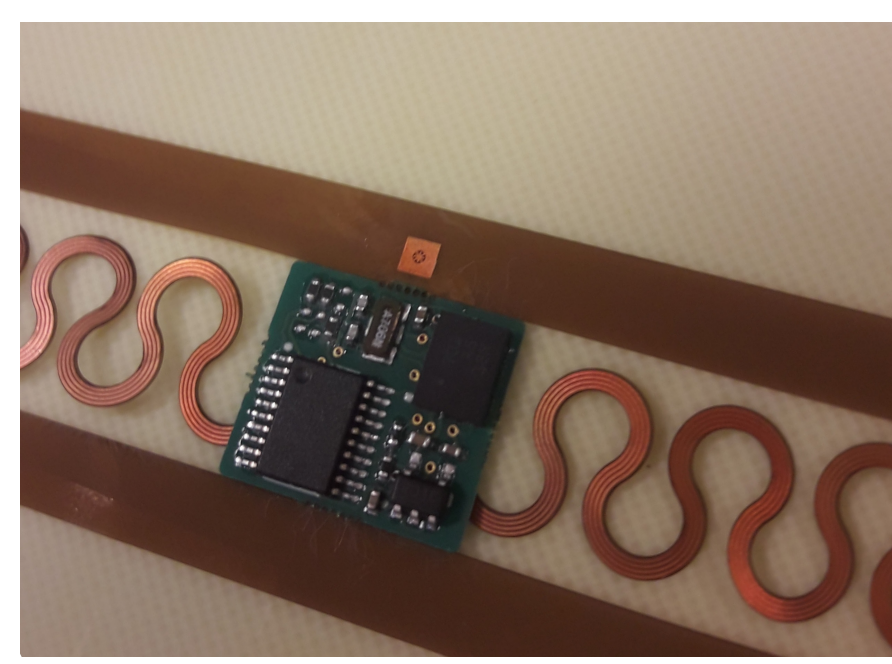

Fig. 3. Detail of the stretchable sensor circuit. The four meandering tracks connect the smart sensor nodes (in picture) to the central controller.

the SMD pads on which components will be mounted are not covered by this adhesive FR0110 material. The SMD areas receive an electroless nickel immersion gold (ENIG) surface finish. The functional flex structure is routed out of the production panel by use of a laser. Small $(12.5 \mathrm{~mm} \times 14 \mathrm{~mm})$ sensor node boards were created to accommodate for the BNO055 IMU sensor. These boards are $200 \mu \mathrm{m}$ thin FR4 boards and also hold all the necessary electronics for voltage regulation, IMU peripherals and $\mathrm{I}^{2} \mathrm{C}$ address control. The sensor boards were soldered manually onto the flexible bus circuit (See Figure 3). This assembled circuit was used to build up the stack as described in the section above. The stack was vacuum laminated using the following parameters. A pressure of $10 \mathrm{bar}$ is applied. The starting temperature is $100^{\circ} \mathrm{C}$ which is ramped up to $175^{\circ} \mathrm{C}$ in 17 minutes and cools finally down to $60^{\circ} \mathrm{C}$. In order to thermoform the laminated stack, it is heated up to $200^{\circ} \mathrm{C}$ and manually thermoformed over the mold into the desired shape.

\section{E. Issues}

The samples were tested for functionality (communication with the sensors) after lamination, after thermoforming, and under load (light, repetitive stresses on the formed shape). It was noticed that after lamination a large part of the samples were unresponsive. The functioning samples were still responsive after thermoforming, but quickly failed under load.

The latter was attributed to cracking of the copper tracks. There is a mechanical weak point where the copper track meets the relatively rigid FR4 board. Repetitive movements, albeit small, damage the copper at that level up to failure. The flexible circuit needed to be redesigned to resolve that problem.

A bigger issue was the fact that a large part of our sensors didn't seem to survive the lamination process itself. The pressure and temperature of the vacuum lamination process

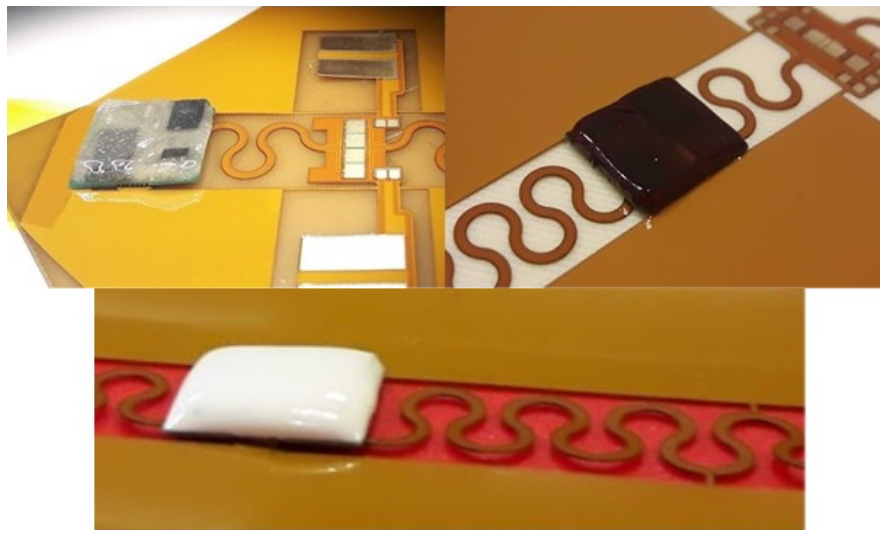

Fig. 4. The smart sensor nodes were encapsulated in epoxy to increase the yield during the lamination process.

are fixed, so clearly some preprocessing is needed to mitigate this problem.

\section{F. Mitigation}

To increase the yield during lamination, the sensor boards themselves were reinforced with various types of glob topping and underfill. For all samples, underfill material Namics XS8455-48 was used below the electronic components on the thin PCB. This to reduce pressure on the component leads when pressure is applied during lamination or walking with the AFO. To further protect the electronic components on the PCB, a glob top was applied. First we tested the use of a silicone material (Dow Corning Silastic E), but it became clear that the silicone was torn after lamination of the circuit between PP.

Then the use of an epoxy Epotek 353 ND - T1 was evaluated, which is manually applied using a 3D printed plate with a cavity. The epoxy is cured at $120^{\circ} \mathrm{C}$ during 10 minutes. After curing, this material is hard and should protect the electronics during lamination. However, sensors were still destroyed after the lamination step.

The lamination process itself was also examined more closely. This showed that the PP sheets were not heated before applying pressure, but only during. While in a standard lamination process, this does not raise any problems, the situation changes when active sensor boards are pressed between two sheets. These boards have chips and other components that protrude from the board. If the PP sheets were to be heated before applying pressure, the PP would flow around these components. On the other hand, if pressure is applied without preheating, these protruding components would receive the brunt of the force. It is estimated that during the process, these components would receive a staggering 13 tonnes of pressure. To remove this initial pressure from the sensor boards during the lamination process, small indentations were milled in the PP sheets at the height of the sensor boards.

With these adjustments, yield of the lamination process was drastically increased, and after thermoforming the resulting part seemed more robust in use. 


\section{Results AND APPLICATION}

The process for laminating a sensor system in PP was developed as part of research towards an Intelligent Integrated Ankle-Foot Orthosis (I2AFO). An AFO is a brace that is worn on the lower leg and foot, to support the ankle, hold the foot and ankle in the correct position, correct foot drop, etc. It can be used for revalidation, but also to improve walking of children by limiting movement and supporting weak muscles. In the project consortium, VIGO (Wetteren, Belgium), with world class expertise in orthopedic solutions, was present as one of the industrial partners. The AFO manufacturing process consists of draping a heated PP sheet over a mold, that was designed for a specific customer.

Embedding a sensor system inside such an AFO would allow the clinicians to get a better understanding of the effectiveness of the AFO. The data from the embedded IMUs would allow for gait analysis in an everyday, real environment as opposed to in a lab. To achieve this, a lot more than an embedded sensor system is needed. A custom central controller was developed (the $\mathrm{I}^{2} \mathrm{C}$ master) to read out the sensor data and send it through Bluetooth to a smartphone, where the data is collected, stored, and visualized. Machine learning was employed to analyse the data and map it to gait profiles. Figure 5 shows a finalized I2AFO.

With the modified vacuum lamination process, we were able to consistently provide sheets with the embedded sensor circuit to VIGO, allowing them to create a series of I2AFOs to be tested by volunteers.

\section{CONCLUSION}

In this paper we presented an approach to embed a movement sensor system in a PP sheet, to be used in a thermoforming process. The system remained functional after thermoforming and a functional intelligent AFO was created. However, repeated use of the AFO and the inferred repetitive stresses in the material, showed that the overall robustness could still be improved.

\section{ACKNOWLEDGEMENTS}

This research was part of the ICON project 'Intelligent Instrumented Ankle-Foot Orthosis' (I2AFO), project number HBC.2016.0654, funded by imec and Flanders Innovation and Entrepreneurship (VLAIO). We would also like to extend our gratitude to VIGO and ACB for their cooperation.



Fig. 5. A finalized I2AFO. Three embeddded IMUs are read out by an external central microcontroller, which transmits the sensor data wirelessly through Bluetooth. 